\title{
BMJ Open Efficacy of acupuncture treatment for chronic spontaneous urticaria: study protocol for a randomised controlled trial
}

\author{
Qianhua Zheng (D) , ${ }^{1}$ Hui Zheng (D) , ${ }^{1}$ Siyuan Zhou, ${ }^{1}$ Yunzhou Shi (D) , ${ }^{1}$ \\ Leixiao Zhang (1) , 'Xianjun Xiao, ${ }^{1}$ Wei Zhang, ${ }^{2}$ Li Zhou, ${ }^{3}$ Ying Huang, ${ }^{4}$ \\ Mingling Chen (D) , ${ }^{4}$ Feng Zhong, ${ }^{2}$ Chuan Wang, ${ }^{3}$ Ying Li (D) ${ }^{5}$
}

To cite: Zheng Q, Zheng $\mathrm{H}$, Zhou S, et al. Efficacy of acupuncture treatment for chronic spontaneous urticaria: study protocol for a randomised controlled trial. BMJ Open 2022;12:e045027. doi:10.1136/ bmjopen-2020-045027

- Prepublication history for this paper is available online To view these files, please visit the journal online (http://dx.doi. org/10.1136/bmjopen-2020045027).

QZ and $\mathrm{HZ}$ contributed equally.

Received 17 November 2020 Accepted 15 October 2021

Check for updates

(c) Author(s) (or their employer(s)) 2022. Re-use permitted under CC BY-NC. No commercial re-use. See rights and permissions. Published by BMJ.

For numbered affiliations see end of article.

Correspondence to

Professor Ying Li;

liying@cdutcm.edu.cn

\section{ABSTRACT}

Introduction Chronic spontaneous urticaria (CSU) is a troublesome dermatological problem that can have a significant impact on quality of life. Previous studies have indicated that acupuncture may be beneficial for patients with CSU. However, well-designed studies determine the effects of acupuncture on CSU are rare. The aim of this study is to investigate the efficacy and safety of acupuncture treatment for patients with CSU.

Methods and analysis This study is designed as a multicentre, parallel, three-arm, randomised, shamcontrolled trial. A total of 330 patients diagnosed as CSU will be randomly allocated into three groups: the verum acupuncture group, the sham acupuncture group and the waiting-list control group in a 1:1:1 ratio. Patients in the verum and sham acupuncture groups will receive 16 treatment sessions over 4 weeks, while patients in the waiting-list control group will not receive any acupuncture treatment. The primary outcome is the changes of weekly urticaria activity scores at the end of treatment. Secondary outcomes include itching severity measurement,

Dermatology Life Quality Index, Hamilton Depression Scale, Hamilton Anxiety Scale, Pittsburgh Sleep Quality Index and serum total lgE level. Adverse events will be recorded during the study observation period. All patients who are randomised in this study will be included in the intentionto-treat analysis.

Ethics and dissemination Ethical approval of this study has been granted by the Sichuan Regional Ethics Review of Committee on Traditional Chinese Medicine (TCM) (ID: $2019 \mathrm{kl}$-006), the Medical Ethic Committee of the First Hospital of Wuhan (ID: (2019) number 7)) and the Medical Ethics Committee of the First Hospital of Hunan University of TCM (ID: HN-LLKY-2019-017-01/03) in three clinical centres in China, respectively. The results will be disseminated through peer-reviewed journals. Trial registration number ChiCTR1900022994.

\section{INTRODUCTION}

Chronic urticaria (CU) is a dermatological condition defined as the recurrence of itchy wheals and/or angioedema daily, or almost daily, lasting at least 6 weeks. ${ }^{1}$ The estimated prevalence of $\mathrm{CU}$ ranges from $0.5 \%$ to $1.0 \%$

\section{Strengths and limitations of this study}

- This is the first study to provide information about the efficacy and safety of acupuncture treatment for patients with chronic spontaneous urticaria by comparing verum versus sham acupuncture and waiting list.

- The risk of bias will be reduced by rigorous methodology, including adequate randomisation, quality control and the use of blinded outcome assessors and statisticians.

- Eligible participants will be strictly recruited and screened in three tertiary A hospitals in China, the results may not apply to patients in other countries.

- Because of the nature of acupuncture, unblinded participants and treatment providers are likely to bring bias and be influencing the results.

in the general population. ${ }^{2}$ Most of the affected patients are between 30 and 50 years old, and there is a significantly higher incidence in women than in men. ${ }^{34}$

There are two types of CU: chronic spontaneous (idiopathic) urticaria (CSU) and chronic inducible urticaria (CIU). The main difference between the two is the former type occurs without identifiable triggers. ${ }^{12}$ Several studies reported that $\mathrm{CU}$ has a significant negative impact on patients' quality of life $(\mathrm{QoL})^{15}$ in terms of sleep and daily activities such as work, study, recreation and social interaction. ${ }^{5-8}$ The decrease in QoL reported by patients with $\mathrm{CU}$ was comparable to that of patient with coronary artery disease ${ }^{9}$ and psoriasis. ${ }^{10}$ The high rates of absenteeism from work and school because of sick leave and medical care, ${ }^{7}$ as well as for various autoimmune $^{11}$ and psychological ${ }^{512}$ comorbidities, directly and indirectly increase the economic burden for patients with $\mathrm{CU}{ }^{8}$ 
According to the consensus on the treatment options for CU, non-sedative H1-antihistamine is the first-line medication, and the doses could increase up to fourfold in refractory cases as the second-line medication. ${ }^{1}$ According to the 2017 version of guideline, omalizumab and cyclosporine A are recommended as the third-line and fourth-line therapy, respectively, for who fail to respond to H1-antihistamine. ${ }^{1}$ Although omalizumab has been reported effective and safe for $\mathrm{CU}$ in recent studies, ${ }^{13} 14$ the high cost are burdensome for most patients. Because of the higher incidence of adverse effects, ciclosporin A cannot be recommended as standard treatment. ${ }^{15}$

Acupuncture, which has been used for more than 3000 years, has been widely applied in clinical practice to treat dermatological problems. ${ }^{16}{ }^{17}$ According to the literatures, acupuncture has been applied for a long time in China to treat urticaria. A systematic review included six randomised controlled trials (RCTs) studying the effectiveness and safety of acupuncture treatment for patients with $\mathrm{CU} .{ }^{18}$ Although the evidence supported that patients with $\mathrm{CU}$ may benefit from acupuncture treatment, the poor methodology of the RCTs, particularly in choosing controls and outcomes for assessing the symptoms and QoL, makes the evidence less confirmative.

To counter these deficiencies, a prospective, parallel, three-arm, randomised, sham-controlled trial with a large sample size will be carried out to investigate the efficacy and safety of acupuncture treatment for patients with CSU, comparing them with sham acupuncture and waiting-list control groups. We hope that the findings from this study will provide solid evidence for the efficacy of acupuncture treatment for CSU.

\section{METHODS \\ Study design}

This is a multicentre, parallel, three-arm, randomised, sham-controlled trial to testify the efficacy of acupuncture treatment for patient with CSU. This trial will be performed at three settings in China: the First Teaching Hospital of Chengdu University of Traditional Chinese Medicine (TCM), Wuhan Hospital of Traditional Chinese and Western Medicine, the First Hospital of Hunan University of Chinese Medicine. Eligible patients with CSU will be included in our study and randomly allocated into three groups: the verum acupuncture group, the sham acupuncture group and the waiting-list group (no treatment) in a 1:1:1 ratio. The total observation period is 9 weeks, including a 1-week baseline, 4-week treatment phase and 4-week follow-up phase. The flowchart of this study is shown in figure 1, and the time points of assessment are shown in table 1 .

\section{Exclusion criteria}

Patients will be excluded if they meet any of the following aspects:

1. Diagnosed as acute urticaria or CIU.
2. Having difficulties in expressing their symptoms clearly such as severe mental disorders or cognitive impairment.

3. Currently having other serious chronic problems, such as cardiovascular, hepatic, renal, gastrointestinal, haematological, infectious diseases or malignant tumours.

4. Having contraindications to acupuncture treatment such as bleeding tendency or local infection at acupoints.

5. Pregnant or breastfeeding.

\section{Recruitment process}

Participants will be recruited primarily from the Dermatology Department in the First Teaching Hospital of Chengdu University of TCM, Wuhan Hospital of Traditional Chinese and Western Medicine and the First Hospital of Hunan University of Chinese Medicine in China. Research assistants will be sent to help screen participants in these hospitals. In addition, potential participants will be recruited through advertisements and survey questionnaires to the public in the hospitals, communities and campuses.

\section{Written informed consent}

In the written informed consent, all participants will be informed that they will be randomly allocated into verum acupuncture, sham acupuncture or waiting-list control groups, with the possible benefits and risks. They will voluntarily sign the informed consent before participating in this study and will be free to withdraw at any time during the study period.

\section{Randomisation, allocation concealment and blinding}

Patients with CSU who meet the eligibility criteria will be randomly assigned to three groups: the verum acupuncture group, the sham acupuncture group or the waitinglist control group in a ratio of $1: 1: 1$. The randomisation sequence will be generated by a computer system in the clinical evaluation centre of China Academy of Chinese Medical Science (CEC-CACMS) with a completely randomised design. The clinical research coordinator in each hospital will be responsible for requesting the randomisation sequence and group assignments through the internet system or mobile phone message. The randomisation sequence will be concealed in the server of CECCACMS until this study finishes participant enrollment, observation and data collection.

In this study, we will apply a type of sham acupuncture device to mask verum and sham acupuncture between patients from the two acupuncture treatment groups, and each patient will be treated in a separate room. A blinding assessment will be performed between patients in acupuncture groups. However, because of the nature of acupuncture, it is difficult to achieve masking between acupuncture groups and the waiting-list control group. Thus, the outcome assessors and statisticians who are responsible for statistical analysis will be masked with 


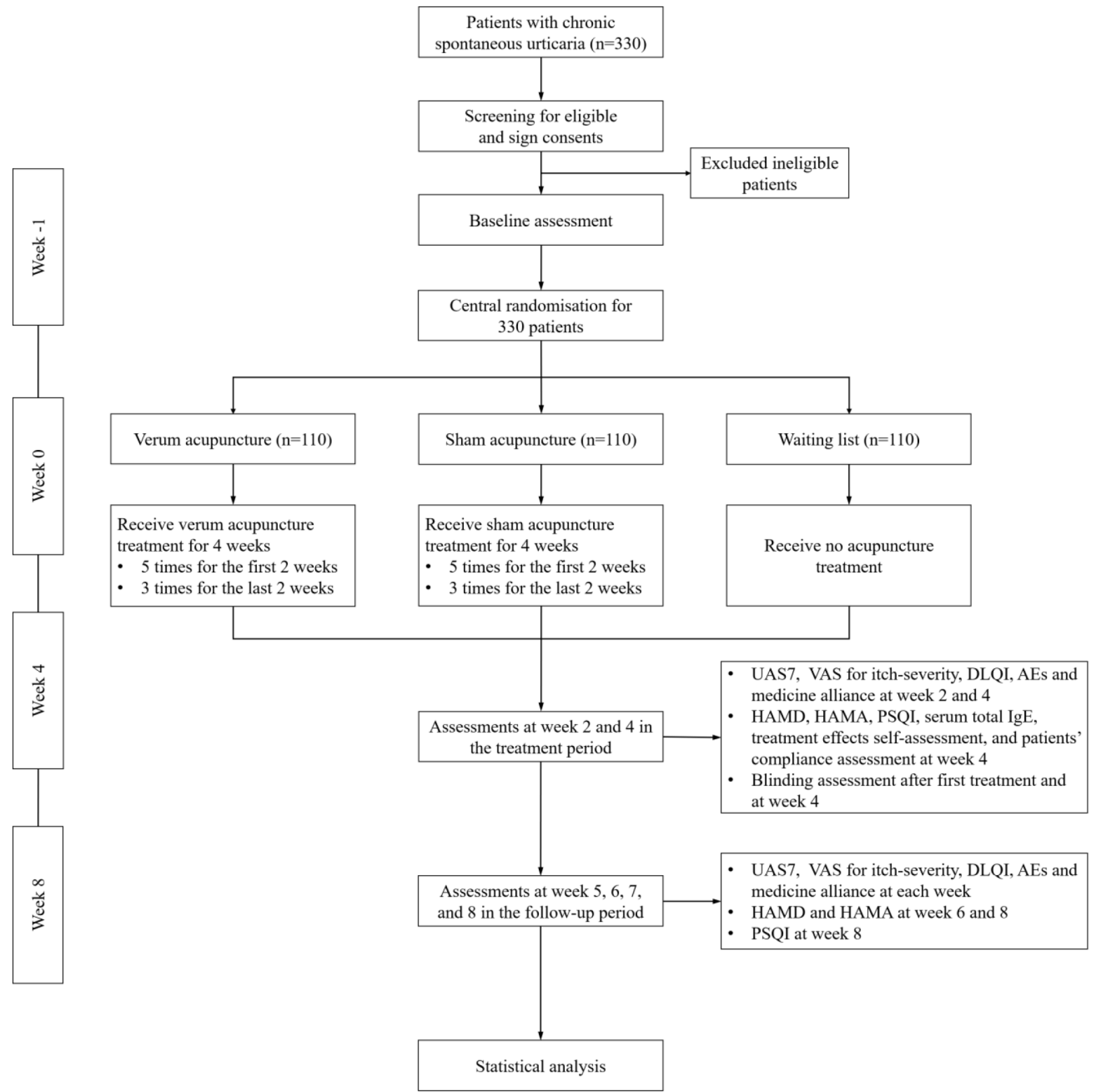

Figure 1 Trial flow chart. DLQI, Dermatology Life Quality Index; HAMA, Hamilton Anxiety Scale; HAMD, Hamilton Depression Scale; IgE, immunoglobulin E; PSQI, Pittsburgh Sleep Quality Index; UAS7, Urticaria Activity Score for 7 consecutive days; VAS, visual analogue scale.

respect of group assignments in the process of performing this study and data analysis.

\section{Interventions}

All participants will receive 16 sessions verum acupuncture treatment or sham acupuncture treatment over 4 weeks, including five consecutive sessions for the first 2 weeks with 2 days off, and three sessions for the last 2 weeks performed every other day. Participants in the waiting-list control group will not receive any acupuncture treatment during the observation period. If the symptoms cannot be relieved or get worse during the observation period, participants will be allowed to take loratadine tablets (Clarityne, Bayer Shanghai, China) $10 \mathrm{mg} /$ day for emergency use, which are second-generation antihistamine drugs, but no steroids and immunosuppressive drugs will be prescribed. If patients fail to respond to loratadine, Ebastine (Sudi, Lianhuan Pharmacy, Jiangsu, China) $10 \mathrm{mg} /$ day will be an alternative. The details of medication use will be documented in the case report form (CRF). If a patient uses steroids and immunosuppressive drugs during the observation period, he or she will be excluded from this study. Sterile disposable acupuncture needles 25-40 mm in length and $0.25 \mathrm{~mm}$ in diameter (Hwatuo, Suzhou, China), sterile disposable blunt and retractable needles (AcuPrime, Exeter, UK) ${ }^{19}$ and Park Sham Placebo Acupuncture Device (PSD) (Dong-bang Acupuncture, Seoul, Korea $)^{20}$ will be used in this study for verum and 
Table 1 Time points of treatment assessment

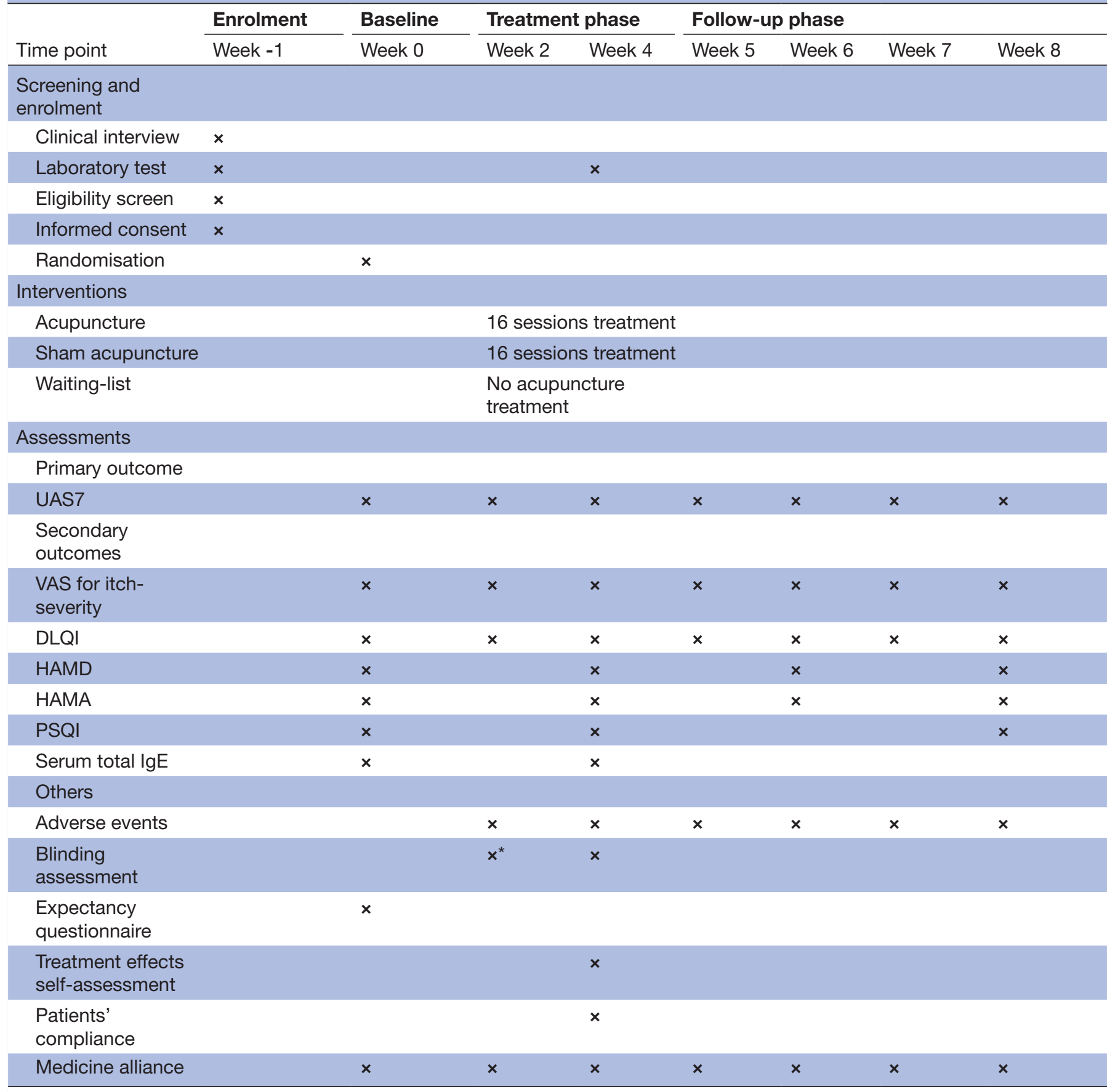

*Assessed after the first session of acupuncture treatment.

DLQI, Dermatology Life Quality Index; HAMA, Hamilton Anxiety Scale; HAMD, Hamilton Depression Scale; IgE, imunoglobulin E; PSQI, Pittsburgh Sleep Quality Index; UAS7, Urticaria Activity Score for 7 consecutive days; VAS, visual analogue scale.

sham acupuncture treatments as well as blinding the treatments from each other. All acupuncturists participated in three special training classes during March and July 2019, to learn how to locate the acupoints and nonacupoints, to use the sham acupuncture device and to insert and manipulate the needles. Acupuncture treatment will be performed by acupuncturists who passed an examination following this training.

\section{Verum acupuncture group}

Patients in the verum acupuncture group will receive treatment with real acupuncture needles inserted at the specific acupoints. In accordance with the textbooks of TCM, literature research and opinions from acupuncture experts, patients in the verum acupuncture group will have real needles inserted at the following points: LI11 (Quchi), SP10 (Xuehai), ST36 (Zusanli), ST25 (Tianshu), 
Table 2 Acupoints used in the verum acupuncture group

\begin{tabular}{ll}
\hline Acupoint & Location \\
\hline LI11 (Quchi) & $\begin{array}{l}\text { On the lateral end of the transverse cubital crease, at the midpoint between LU5 (Chize) with the lateral } \\
\text { epicondyle of humerus (needled bilaterally) }\end{array}$ \\
$\begin{array}{l}\text { On the prominence of the medial head of the quadriceps femoris, } 2 \text { cun above the medio-superior } \\
\text { border of the base of patella (needled bilaterally) }\end{array}$ \\
SP6 (Sanyinjiao) & $\begin{array}{l}\text { On the medial side of the shank, } 3 \text { cun above the medial malleolus, by the posterior border of the tibia } \\
\text { (needled bilaterally) }\end{array}$ \\
ST36 (Zusanli) & $\begin{array}{l}\text { On the anterior lateral side of the shank, } 3 \text { cun below ST35 (Dubi), one horizontally placed finger } \\
\text { distance lateral to the anterior border of the tibia (the middle finger) (needled bilaterally) }\end{array}$ \\
ST25 (Tianshu) & $\begin{array}{l}\text { On the middle portion of the abdomen, } 2 \text { cun lateral to the central of the navel (needled bilaterally) } \\
\text { On the anterior media line of the upper abdomen, } 4 \text { cun above the navel }\end{array}$ \\
HT7 (Shenmen) & $\begin{array}{l}\text { On the wrist, at the ulnar end of the transverse crease of the wrist, in the depression on the radial side } \\
\text { of the tendon of the flexor carpi ulnaris (needled bilaterally) }\end{array}$ \\
\hline
\end{tabular}

SP6 (Sanyinjiao), HT7 (Shenmen) bilaterally and CV12 (Zhongwan). Details of the location of acupoints are shown in table 2 and figure 2. First, the pedestal of PSD will be adhered to the skin at each acupoint. The real acupuncture needles with tips will be inserted into the tube and then penetrate the skin. Then, according to the location of points and the individual's condition, needles will be positioned at the appropriate depth and angle. During the needle retention, the pedestal and tube of devices will not be removed to retain the blinding of the type of needle application. Manipulations of twirling, lifting and thrusting will be performed on each needle to achieve
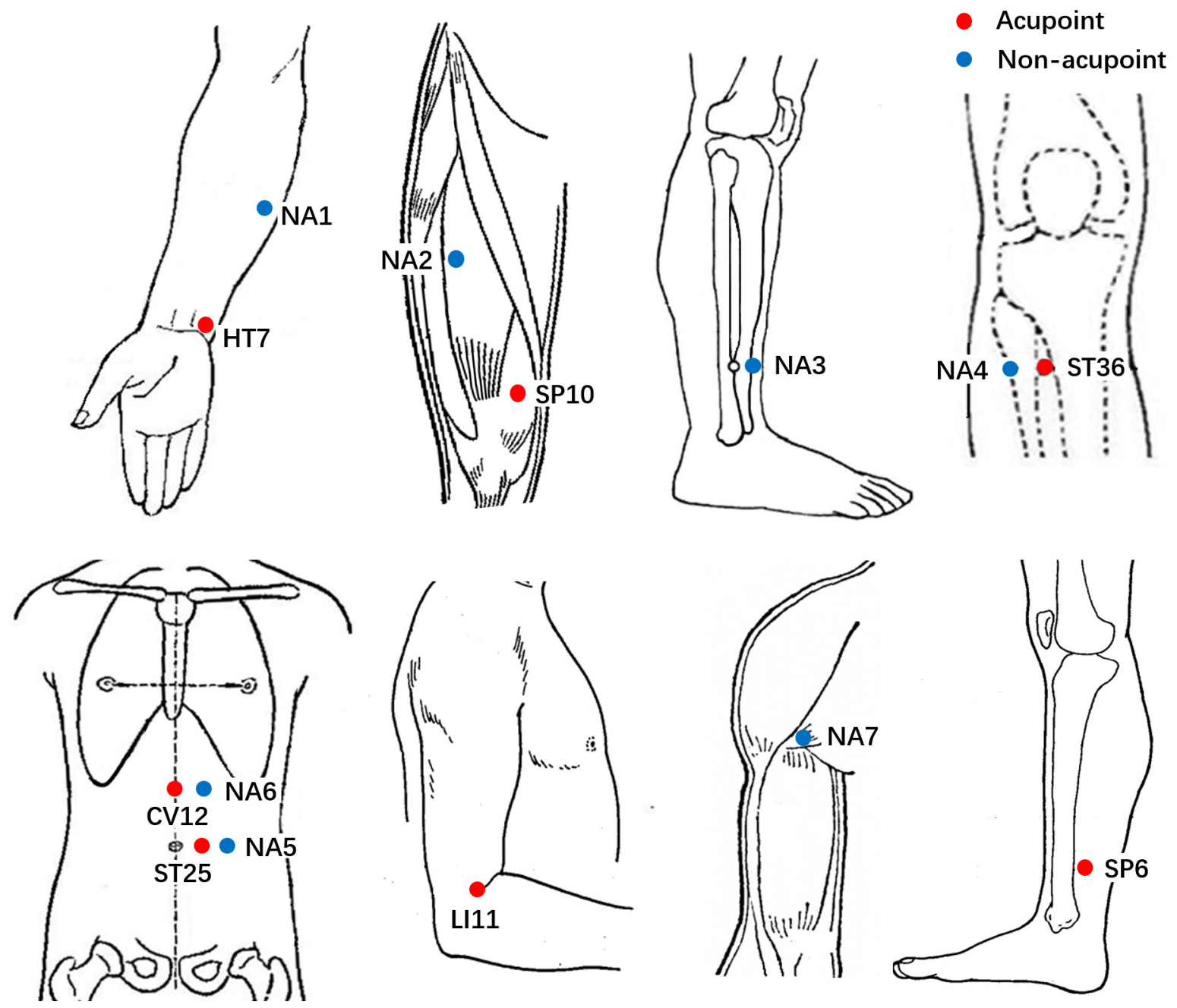

Figure 2 Location of acupoints and non-acupoints. 
Table 3 Non-acupoints used in the sham acupuncture group

\begin{tabular}{ll}
\hline Non-acupoint & Location \\
\hline Non-acupoint 1 & $\begin{array}{l}\text { On the radial side, the midpoint between the medial epicondyle of humerus and the styloid process of } \\
\text { ulna (needled bilaterally) }\end{array}$ \\
Non-acupoint 2 & $\begin{array}{l}\text { On the thigh, 0.8 cun medial to the midpoint of the line linking the anterior superior iliac spine and the } \\
\text { lateral end of the base of patella (needled bilaterally) }\end{array}$ \\
Non-acupoint 3 & $\begin{array}{l}\text { On the lateral side of the lower leg, 3 cun above the tip of external malleolus, between Stomach } \\
\text { Meridian of Foot-Yangming and Gallbladder Meridian of Foot-Shaoyang (needled bilaterally) }\end{array}$ \\
Non-acupoint 4 & $\begin{array}{l}\text { On the lateral side of the lower leg, 1 cun lateral to ST36 (Zusanli), between Stomach Meridian of Foot- } \\
\text { Yangming and Gallbladder Meridian of Foot-Shaoyang (needled bilaterally) }\end{array}$ \\
Non-acupoint 5 & $\begin{array}{l}\text { On the middle of abdomen, } 1 \text { cun lateral to ST25 (Tianshu), the midpoint between ST25 and SP15 } \\
\text { (Daheng) (needled bilaterally) }\end{array}$ \\
Non-acupoint 6 & $\begin{array}{l}\text { On the middle of abdomen, 1.2 cun lateral to CV12 (Zhongwan) (applied on body side alternatively per } \\
\text { each treatment session) }\end{array}$ \\
Non-acupoint 7 & $\begin{array}{l}\text { On the medial anterior border of the upper arm, the junction of the deltoid and the biceps brachii } \\
\text { (needled bilaterally) }\end{array}$ \\
\hline
\end{tabular}

the Deqi sensation. The treatment will last $30 \mathrm{~min}$, and during the needle retention period, the needles will be manipulated by manual twirling, lifting and thrusting every $10 \mathrm{~min}$ to maintain the Deqi sensation.

\section{Sham acupuncture group}

Patients in the sham acupuncture group will receive non-penetrating sham acupuncture at non-acupoints. In this group, non-penetrating sham acupuncture and nonacupoints will serve as controls for manual puncturing stimulation and specific acupoints, respectively. Sham acupuncture will be administrated by PSD with blunt and retractable needles at non-acupoints. Details of the location of non-acupoints, which are different than the conventional acupoints, located along the meridians are shown in table 3 and figure 2. After the acupuncturist inserts the needle into the tube, the needle will retract into the handle when the blunt tip touches the skin. There will be no Deqi sensation. The manipulation techniques will be the same as in the verum acupuncture group with 30 min duration without needle penetration. In China, most people have experienced an acupuncture treatment. Although we will exclude patients who have received acupuncture treatment 4 weeks prior to the study and use the sham device, there is still high possibility that patients will distinguish the sham from verum acupuncture. Therefore, patients in the different acupuncture groups will be placed in separate room for receiving treatment and be asked to guess which type of acupuncture they receive after treatment to assess the blinding effects.

\section{Waiting-list control group}

Patients in the waiting-list control group will not receive any acupuncture treatment in the 9-week observation period. After the end of follow-up period, they will be offered 10 free sessions of acupuncture treatment with the same treatment protocol applied as the patients in verum acupuncture group as compensation.

\section{Outcome measures}

Primary outcome measurement

The primary outcome is the changes of weekly urticaria activity scores (UAS7), which will be used for patient selfevaluation of the severity of urticaria once daily for 7 consecutive days. UAS have been widely used to measure the symptoms of urticaria. It scores the number of wheals and the severity of pruritus on a 4-point scale of 0 to 3 . Patients in this study will be trained to use this scale for self-evaluation and required to assess their symptoms in every 24 hours for 7 days a week before each clinic visit. They will be required to fill in a 7-day urticaria diary and give it to assessors when coming to the clinic. The scores of UAS7 are the sum of daily UAS scores, ranging from 0 to 42 . The higher the scores, the worse the condition. UAS7 is a unified and simple scoring system that has recommended by the guidelines and has been validated. ${ }^{1-23}$ UAS7 will be measured at baseline, week 2 and week 4 in the treatment phase, and weekly in the follow-up phase (visit points 2-8). The schedule of measurement and visit points are shown in table 1 . The end point of this study is the change from baseline in UAS7 scores at the end of treatment (visit point 4).

\section{Secondary outcome measurement}

The secondary outcomes of this study include itching severity measurement, Dermatology Life Quality Index (DLQI), Hamilton Depression Scale (HAMD), Hamilton Anxiety Scale (HAMA), Pittsburgh Sleep Quality Index (PSQI) and serum total IgE level. The schedule of measurements and visits for each outcome are shown in table 1. All patients are required to fill in a 7-day urticaria diary. Patients will be reminded to fill these diaries by short message, phone calls, e-mails and other social software, such as WeChat.

Itch-severity measurement

Itching is one of the troublesome symptoms that has a negative influence on CSU patients' QoL and worsening 
patents' well-being. ${ }^{24}{ }^{25}$ In this study, a $0-10 \mathrm{~cm}$ visual analogue scale will be used to assess itching severity of CSU (0 for no itching to 10 for intolerable itching). Itching severity will be measured daily by patients themselves at baseline, week 2 and week 4 in the treatment phase, and weekly in the follow-up phase (visit points 2-8). The total scores are the sum scores of daily scores for 7 consecutive days.

\section{Quality of life}

Available data indicate that CSU markedly affects patients' QoL. ${ }^{58}$ A related questionnaire evaluating QoL of patients is recommended by the guideline. ${ }^{1}$ The DLQI is a professional questionnaire for evaluating the QoL impacted by dermatological problems, which has been validated for patients with CSU. ${ }^{23}{ }^{26}$ It consists of 10 items in six subscales: symptoms and feelings, daily activities, leisure, personal relationships, work and school and treatment. ${ }^{27}$ Each item is scored as $0,1,2$ or 3 according to the different degrees that QoL is impaired. The total scores of DLQI vary between 0 and 30 . The higher scores the patients' scores, the more QoL is impaired. We will use the Chinese simplified version downloaded from official website (wwwdermatologyorguk.). DLQI will be measured at baseline, week 2 and week 4 in the treatment phase, and weekly in the follow-up phase (visit points 2-8).

\section{Psychological condition}

Psychological disorders, such as anxiety and depression, are commonly found in CU patient group. ${ }^{28} \mathrm{~A}$ positive correlation between the severity of urticaria and depression or anxiety has been reported. ${ }^{29}$ Additionally, the severity of psychiatric disease indicated a worse QoL in CSU patients. ${ }^{30}{ }^{31}$ Therefore, the HAMD and HAMA will be used to assess patients' psychological status in this study. HAMD and HAMA will be measured at baseline, at the end of treatment, week 6 and week 8 in the follow-up phase (visit points 2, 4, 6 and 8).

\section{Sleep quality}

Patients with CSU commonly report sleep disturbances or insomnia because of hives, itching or both. ${ }^{28}{ }^{32}$ Sleep problems may also be a predisposing factor for $\mathrm{CSU}^{33}$ Poor sleep contributed to fatigue and damaged physical and emotional well-being. ${ }^{2}$ The PSQI is a self-rated questionnaire to assess sleep quality and disturbance that can be used both in clinical practice and research studies for patients with psychiatric or sleep problems and even among the general population. ${ }^{34}$ It contains 19 items in seven subdomains, including subjective sleep quality, sleep latency, sleep duration, habitual sleep efficiency, sleep disturbances, use of sleeping medication and daytime dysfunction. ${ }^{34}$ The sum scores of each item range from 0 to 21. The higher the patients' scores, the worse sleep quality they experience. PSQI will be measured at baseline, at the end of treatment and week 8 in the follow-up period (visit points 2, 4 and 8).
Serum total lgE level

It was reported that the serum IgE level of patients with $\mathrm{CU}$ was higher than in healthy controls ${ }^{35}$ and it was decreased with acupuncture treatment. ${ }^{35}{ }^{36}$ Serum IgE will be examined to detect the allergic and immune status of participants. This test will be performed before randomisation and at the end of treatment (visit points 2 and $4)$.

\section{Patients' expectations about acupuncture treatment}

Patient will fill out an acupuncture expectancy questionnaire before the first acupuncture treatment, which aims to detect whether a patient's expectations will impact acupuncture outcomes. This questionnaire includes seven items. Each of them is rated on a 4-point Likert scale.

\section{Treatment effect self-assessment}

Overall treatment satisfaction will be assessed at the end of treatment (visit point 4) by a 4-point Likert scale. Patients will be asked 'How were your symptoms of urticaria during the past week in comparison with the baseline period?' The answers will include 'cured', 'improved', 'not changed' and 'aggravated'.

\section{Blinding assessment}

When we recruit and screen eligible participants, patients will be informed that they will have an equal chance of receiving traditional acupuncture, acupuncture-like stimulation treatment or no acupuncture treatment. Patients in verum or sham acupuncture groups will be asked to guess what type of acupuncture they receive after the first and the last session of acupuncture treatment to test the patient-blinding effects. The question is 'Which style acupuncture do you think you have received?' The patients will be provided with three options to answer this question: traditional acupuncture, acupuncture-like stimulation or uncertain.

\section{Patients' compliance assessment}

Patients' compliance will be recorded at the end of the treatment (visit point 4). The drop outs and reasons will be recorded in the 9-week observation period.

\section{Use of medication}

Self-reported use of additional medication or healthcare resources to control CSU in the observation period will be required to be recorded in the CSU diary.

\section{Safety assessments}

Safety will be assessed by routine blood tests, renal function as well as liver function tests. These indicators will be measured in the screening period and at the end of treatment (visit point 4). Adverse events (AEs) caused by acupuncture treatment, such as bleeding, pain, haematoma, fainting, local infection and so on, are to be recorded. Serious AEs related to the interventions will be reported to the principal investigator and documented in the CRF and the affected participants will be withdrawn 
from the study. Treatment or rescue applications will be provided in a timely manner. In the assessment period, researchers will assess the possible relationship between AEs and the study as well as the combined medications.

\section{Patients and public involvement}

Patients and the public are not involved in the design or conduct of the study or the outcome measures, and no attempt will be made to assess the burden of the intervention on the patients themselves. The results of this study will be disseminated to study participants via the website of our hospitals.

\section{Sample size}

Before undertaking this study, we performed a pilot study with a small sample size to determine the efficacy of acupuncture for CSU. The protocol has been published in a peer-reviewed journal. ${ }^{37}$ In this pilot study, the changes of UAS7 scores in verum acupuncture and sham acupuncture were 16 and 12, respectively (the results are still unpublished). The difference of scores between two groups was 4 . Therefore, we assume that the verum acupuncture and sham acupuncture will reduce the UAS7 scores as in the preliminary study. We expect the population SD to be 10 with effect size (Cohen's d) 4. One hundred patients per group would be needed as calculated by the $\mathrm{G}$ power (V.3.0.10) at a two-sided significance level of $5 \%$ and power of $80 \%$ in a 1:1:1 ratio. Estimating a $10 \%$ drop out rate, a total of 330 patients (110 patients per group) will need to be enrolled in this study.

\section{Data management}

All data will be documented by outcome assessors at each site who have been qualified by a special training class before recruitment. The training includes how to fill in the CRF in an accurate and timely manner, how to assess the electronic CRF (eCRF) that is established by the CECCACMS and input the data into the eCRF to double check the accuracy. Standard operation procedures have been established and provided to the assessors and researchers in each clinical trial institution. The data for each participant will be stored in the centre server of the CECCACMS, and only the data monitor is able to check the data. The researchers are unable to modify and acquire data before all participants' enrollment, observation and data collection are finished.

\section{Statistical analysis}

SPSS V.21.0 statistical software (IBM, Armonk, New York, USA) will be used for data analysis. An independent statistician who does not know the group assignments will run the statistical analysis. Continuous variables will be described as mean \pm SD with a $95 \%$ CI in a normal distribution and median (range) in abnormal distribution, while categorical variables will be represented by numbers (percentages). The level of significance will be established at 0.05 in a two-sided test.

All analyses will be performed according to intention-totreat (ITT) protocol. Participants who finish the baseline assessment of primary outcome and receive at least one session of either verum or sham acupuncture treatment will be included in ITT population. The missing data of participants who drop out will be handled by multiple imputations with a package 'Amelia' from the R software (www.r-project. org). Per-protocol population analysis will be also performed, for participants who have finished at least $80 \%$ of the treatment protocol after randomisation.

The primary outcome will be compared by an analysis of covariance (ANCOVA). The ANCOVA will be adjusted by the patients' characteristics and baseline outcomes if there are possible covariates. Pairwise comparisons will be performed by the least significant difference test in a post hoc analysis, if the difference is significant in the global test among three groups (verum acupuncture, sham acupuncture and waiting-list). There are eight visit points of this study. Therefore, repeated measures ANCOVA will be performed to detect the differences between groups at different visit points, and interactions between groups and time points. The secondary outcomes will be analysed in the same model of ANCOVA. Categorial variables will be compared by $\chi^{2}$ test or Fisher's exact test. A paired $\mathrm{t}$ test or Wilcoxon signed-rank test will be used to compare the differences within groups.

\section{Quality control}

All staff participated in special training about the study objectives, study protocol on the treatment strategies and quality control before the study began. Acupuncturists are licensed and have at least 2 years of acupuncture experience in clinical practice. All study documents (such as screening forms, CRFs, treatment records) and treatment material (such as acupuncture needles, PSD, etc) are in locked storage at study sites with limited access. A specified monitor in each site will check the CRFs and records of the acupuncture treatment every week. Every 3 months, members of the quality monitor group will perform a quality control review at each study site and produce a report on the quality of the entire study process. The principal investigator will hold regular meetings to discuss and solve the problems discovered during the observation period.

\section{Patient and public involvement}

Patient priorities, experiences and preferences were not involved in the development of the research question, outcome measures, study design, recruitment or conduct of this study. The results will not be disseminated to study participants. The burden of intervention will not be assessed by trial participants. The results of this study will be disseminated to study participants via the website of our hospitals.

\section{Ethics and dissemination}

The protocol of this study has been approved by the Sichuan Regional Ethics Review of Committee on TCM (Medical Ethics Committee of the Affiliated Hospital of Chengdu University of TCM) (Approval ID: 2019 kl-006), 
the Medical Ethics Committee of the First Hospital of Wuhan (approval ID: (2019) number 7) and the Medical Ethics Committee of the First Hospital of Hunan University of TCM) (Approval ID: HN-LLKY-2019-017-01/03). This study will be performed in accordance with Helsinki declaration and has been registered on the Chinese Clinical Trial Registry (CHICTR) platform. Each patient will be given full and adequate information about the purpose, possible risks and benefits of the study. Written informed consent is reviewed with the patient by the recruiting physician and signed by patients prior to study entry. Personal information will be removed and replaced with a new, unique and unidentifiable identification number in this study, and there will be no personal details in period of dissemination, thus ensuring the confidentiality of the information. The results of this study will be presented in select conferences and scientific meetings and will be published in peer-reviewed journals. For transparency of study results, the raw data set will be available on request to the research team 3 years after the publication of study results.

\section{DISCUSSION}

CSU significantly impairs patients' QoL and leads to a great economic burden for the families as well as society. This multicentre, parallel, three-arm, randomised, shamcontrolled trial will examine the efficacy of acupuncture in treating CSU and provide solid evidence of acupuncture treatment for patients with CSU.

According to the theory of TCM, the main aetiology and pathogenesis of CSU is the disharmony of nutrients and defences, resulting from the wind evil assailing the exterior, accumulated heat in stomach and intestine, blood deficiency and wind-dryness. Therefore, a method of dispelling wind, harmonising nutrients and relieving itching should be used for treating CSU. In accordance with the literature and consultation with experts, we will choose the acupoints located along the Hand-Yangming and Foot-Yangming Meridians as the main acupuncture points (table 2 and figure 2). LI11 and SP10 can disperse wind and clear heat, harmonise the nutrients and defences, cool blood and combine with ST36, ST25, CV12 and SP6 to invigorate spleen, drain dampness and harmonise blood. SP10 is an acupoint with importance for cooling, regulating and nourishing blood, which is commonly used for skin disease. The Yellow Emperor' s Inner Canon records that symptoms of pain, itching, sores and ulcers all result from dysfunction of the heart. Thus, HT7 will be chosen for tranquillising the spirit and relieving itching. For non-acupoints, we will choose the same number of points located on non-meridians as our sham controls (table 3 and figure 2). These non-acupoints are located near the corresponding traditional Chinese acupoints, which could blind patients from differentiating them from the verum acupoints.

Previous studies reported that acupuncture treatment was beneficial for patients with CU. ${ }^{18} 36$ However, results from these studies seem somewhat unreliable because of poor methodology in the following aspects. First, the sample size of previous studies was small ${ }^{18}$ and lacked a rationale for the sample size calculation. Second, to the best of our knowledge, we did not find reports of any RCT applied sham acupuncture, placebo or waiting-list controls in any study of acupuncture for CU after a systematic literature search in databases, such as PubMed, CNKI, Wangfang et al. So, the specific effects of acupuncture for CU have not been well identified so far. Several methods of sham acupuncture controls have been applied in acupuncture studies, but they are controversial. At present, the common types of controls include non-acupoints (stimulation location), different stimulation methods (such as superficial needling, non-penetrating puncturing or sham-laser acupuncture) and a PSD (such as the Streitberger needle) ${ }^{38} \cdot{ }^{20}$ Real needles at non-acupoints with normal depth or superficial puncturing were reported as having some effects in different diseases. ${ }^{39}{ }^{40}$ Stimulation, such as superficial needling, could trigger cutaneous therapeutic effects to relieve dermatological problems. In this study, we choose non-penetrating needles and nonacupoints as controls for real acupuncture stimulation and specific acupoints in accordance with TCM theory, respectively. An ideal placebo should be inert and unable to be distinguished by patients. Because of the nature of acupuncture, a placebo device is necessary for a blinded study. Therefore, we will apply PSD in this study to ensure blinding in the verum and sham acupuncture groups. Third, several studies chose global symptom scores as outcome measurements that were not the internationally acknowledged outcome measurements for CSU. Furthermore, most of them applied cut points or ranking categories, which may decrease the statistical effects compared with analysis of continuous data. In this study, we will apply USA7, which is recommended by the guidelines, and has been validated, ${ }^{123}$ to assess CSU. Fourth, longterm effects of acupuncture have been demonstrated in various disease, such as migraine, ${ }^{41}$ chronic neck pain, ${ }^{42}$ hot flashes ${ }^{43}$ and tinnitus. ${ }^{44}$ Recurrence and persistence are characteristics of CU. ${ }^{1}$ A previous study reported that a lower recurrence rate was observed in the acupuncture group after the end of treatment, when compared with drugs in treating CU. ${ }^{36}$ Therefore, we are interested in the long-term effects of treatment during the follow-up period on urticaria, psychology, sleeping, QoL and so on. Last, the randomisation sequence is generated by a computer, and only authorised persons have access to information about the randomisation sequence, and group assignment will be concealed in the CEC-CACMS until the end of the study to avoid selection bias.

This study has several limitations. First, because of the nature of acupuncture, blinding the acupuncturists or patients between acupuncture treatment and waiting-list control groups is impossible. Additionally, it is impossible to blind acupuncturists while they are performing verum or sham acupuncture treatment. Therefore, independent outcome assessors and statisticians who are masked from 
group assignments will carry out outcome assessments and data analysis in this study. Second, this study is being performed in Chengdu, Wuhan and Changsha, which are the capitals of Sichuan, Hubei and Hunan Provinces in southwestern and central China, respectively. The patients who we include in this study could be not present patients in China with different ethnic nationalities, either the foreigners living in the western countries.

This trial will follow the Consolidated Standards of Reporting Trial reporting guidelines ${ }^{45}$ and Standard for Reporting Interventions in Clinical Trials of Acupuncture recommendations. ${ }^{46}$ We hope the results of this study will provide high-quality evidence for the use of acupuncture for CSU treatment.

\section{Trial status}

This study is currently in the recruitment phase. The first patient was randomised on 27 May 2019, and the study is expected to end in the middle of 2021.

\section{Ethics approval}

This study protocol was approved by Sichuan Regional Ethics Review of Committee of Traditional Chinese Medicine (23 April 2019, approval number 2019 kl-006), the Medical Ethic Committee of the First Hospital of Wuhan (15 May 2019, approval number (2019) number 7) and the Medical Ethic Committee of the First Hospital of Hunan University of TCM) (12 July 2019, approval number HN-LLKY-2019-017-01/03).

\section{Author affiliations}

${ }^{1}$ Acupuncture and Tuina School, Chengdu University of Traditional Chinese Medicine, Chengdu, Sichuan, China

${ }^{2}$ Acupuncture, Tuina and Rehabilitation Department, The First Hospital of Hunan University of Chinese Medicine, Changsha, Hunan, China

${ }^{3}$ Department of Acupuncture and Moxibustion, Wuhan Hospital of Traditional Chinese and Western Medicine, Wuhan, Hubei, China

${ }^{4}$ Dermatology Department, Chengdu University of Traditional Chinese Medicine Affiliated Hospital, Chengdu, Sichuan, China

${ }^{5}$ Graduate School, Chengdu University of Traditional Chinese Medicine, Chengdu, China

Acknowledgements The authors would like to appreciate the contributions of all the physicians, nurses and staff from the Chengdu, Hunan and Wuhan centres. And thanks for the financial support from the National Key Research and Development Program, and technicians working in the CEC-CACMS. The author would like to acknowledge the following people for their help in this study: Prof. Ning Li from the Department of Acupuncture and Moxibustion, Dr. Jingyi Li from the Dermatology Department of West China Hospital of Sichuan University, and Prof. Ji Li from the Department of Acupuncture and Moxibustion of the First Teaching Hospital of Chengdu University of Traditional Chinese Medicine.

Contributors $\mathrm{YL}$ is a principal investigator and conceived of the study. $\mathrm{YL}, \mathrm{HZ}$, SZ and QZ initiated the study design. YL and SZ are monitors of this study. YS, LZ, XX, FZ and CW will carry out the implementation of this study, including participants' recruitment, acupuncture procedure conducting and the case report forms filling in. YL contributed in obtaining research fund. $\mathrm{HZ}$ was responsible for design of statistical analysis. YL, WZ and LZ sought ethical approval. YH and MC provided professional advice for the study design and will be responsible for screening eligible participants. QZ drafted this manuscript. QZ, HZ, YS and YL were responsible for revising the manuscript. All authors read this manuscript and approved the publication of the final manuscript.
Funding This study is supported by the National Key Research and Development Program of the China-Key Project 'Research on Modernisation of Traditional Chinese Medicine-International Cooperation Research on Evaluation of Acupuncture Advantage Disease' (grant numbers 2017YFC1703600 and 2017YFC1703605).

Disclaimer The funding body has no role in the study design ad will not play any role during its execution, analyses, interpretation of the data, or decision on submit the report for publication.

Competing interests None declared.

Patient and public involvement Patients and/or the public were not involved in the design, or conduct, or reporting, or dissemination plans of this research.

Patient consent for publication Not applicable.

Provenance and peer review Not commissioned; externally peer reviewed.

Data availability statement The data can be available by asking the corresponding author of this study after this study complete.

Open access This is an open access article distributed in accordance with the Creative Commons Attribution Non Commercial (CC BY-NC 4.0) license, which permits others to distribute, remix, adapt, build upon this work non-commercially, and license their derivative works on different terms, provided the original work is properly cited, appropriate credit is given, any changes made indicated, and the use is non-commercial. See: http://creativecommons.org/licenses/by-nc/4.0/.

Author note 2019.02.10 study protocol (original protocol:V1.0); 2019.03.02 study protocol (first amendment: V2.0); 2019.04.10 study protocol (second amendment: V3.0). Any important protocol modifications and other changes after the publication of this paper will be updated at the trial registration platform.

\section{ORCID iDs}

Qianhua Zheng http://orcid.org/0000-0001-5489-7034

Hui Zheng http://orcid.org/0000-0002-0494-1217

Yunzhou Shi http://orcid.org/0000-0002-7560-3290

Leixiao Zhang http://orcid.org/0000-0002-8117-6213

Mingling Chen http://orcid.org/0000-0002-8879-0857

Ying Li http://orcid.org/0000-0001-7554-0958

\section{REFERENCES}

1 Zuberbier T, Aberer W, Asero R, et al. The EAACI/GA2LEN/EDF/WAO guideline for the definition, classification, diagnosis and management of urticaria. Allergy 2018;73:1393-414.

2 Maurer M, Weller K, Bindslev-Jensen C, et al. Unmet clinical needs in chronic spontaneous urticaria. A GA2LEN Task force report. Allergy 2011;66:317-30.

3 Wertenteil S, Strunk A, Garg A. Prevalence estimates for chronic urticaria in the United States: a sex- and age-adjusted population analysis. J Am Acad Dermatol 2019;81:152-6.

4 Curto-Barredo L, Archilla LR, Vives GR, et al. Clinical features of chronic spontaneous urticaria that predict disease prognosis and refractoriness to standard treatment. Acta Derm Venereol 2018;98:641-7.

5 Hoskin B, Ortiz B, Paknis B, et al. Humanistic burden of refractory and Nonrefractory chronic idiopathic urticaria: a real-world study in the United States. Clin Ther 2019;41:205-20.

6 Balp M-M, Weller K, Carboni V, et al. Prevalence and clinical characteristics of chronic spontaneous urticaria in pediatric patients. Pediatr Allergy Immunol 2018;29:630-6.

7 Costa C, Rosmaninho I, Guilherme A, et al. Chronic urticaria in the real-life clinical practice setting in Portugal: baseline results from the non-interventional multicentre aware study. Acta Med Port 2019;32:133-40.

8 Maurer M, Abuzakouk M, Bérard F, et al. The burden of chronic spontaneous urticaria is substantial: real-world evidence from ASSURE-CSU. Allergy 2017;72:2005-16.

9 Baiardini I, Giardini A, Pasquali M, et al. Quality of life and patients' satisfaction in chronic urticaria and respiratory allergy. Allergy 2003;58:621-3.

10 Mendelson MH, Bernstein JA, Gabriel S, et al. Patient-Reported impact of chronic urticaria compared with psoriasis in theUnited states. J Dermatolog Treat 2017;28:229-36.

11 Kolkhir P, Borzova E, Grattan C, et al. Autoimmune comorbidity in chronic spontaneous urticaria: a systematic review. Autoimmun Rev 2017;16:1196-208.

12 Barbosa F, Freitas J, Barbosa A. Chronic idiopathic urticaria and anxiety symptoms. J Health Psychol 2011;16:1038-47. 
13 Tonacci A, Billeci L, Pioggia G, et al. Omalizumab for the treatment of chronic idiopathic urticaria: systematic review of the literature. Pharmacotherapy 2017;37:464-80.

14 Giménez-Arnau AM. Omalizumab for treating chronic spontaneous urticaria: an expert review on efficacy and safety. Expert Opin Biol Ther 2017;17:375-85.

15 Vena GA, Cassano N, Colombo D, et al. Cyclosporine in chronic idiopathic urticaria: a double-blind, randomized, placebo-controlled trial. J Am Acad Dermatol 2006;55:705-9.

16 van den Berg-Wolf $\mathrm{M}$, Burgoon T. Acupuncture and cutaneous medicine: is it effective? Med Acupunct 2017;29:269-75.

17 Ma C, Sivamani RK. Acupuncture as a treatment modality in dermatology: a systematic review. J Altern Complement Med 2015;21:520-9.

18 Yao Q, Li S, Liu X, et al. The effectiveness and safety of acupuncture for patients with chronic urticaria: a systematic review. Biomed Res Int 2016;2016:1-7.

19 Park J, White A, Stevinson C, et al. Validating a new non-penetrating sham acupuncture device: two randomised controlled trials. Acupunct Med 2002;20:168-74

20 Park J, White A, Lee H, et al. Development of a new sham needle. Acupunct Med 1999;17:110-2.

21 Młynek A, Zalewska-Janowska A, Martus P, et al. How to assess disease activity in patients with chronic urticaria? Allergy 2008;63:777-80.

22 Hawro T, Ohanyan T, Schoepke N, et al. Comparison and interpretability of the available urticaria activity scores. Allergy 2018;73:251-5

23 Khalil S, McBride D, Gimenez-Arnau A, et al. Weekly urticaria activity score (UAS7) and dermatology life quality index (DLQI) in validation of chronic Spontaneous/Idiopathic urticaria (CSU/CIU) health states. J Allergy Clin Immunol 2015;135:AB131.

24 Tey HL, Wallengren J, Yosipovitch G. Psychosomatic factors in pruritus. Clin Dermatol 2013;31:31-40.

25 Verhoeven L, Kraaimaat F, Duller P, et al. Cognitive, behavioral, and physiological reactivity to chronic Itching: analogies to chronic pain. Int J Behav Med 2006;13:237-43.

26 Lennox RD, Leahy MJ. Validation of the dermatology life quality index as an outcome measure for urticaria-related quality of life. Ann Allergy Asthma Immunol 2004;93:142-6.

27 Finlay AY, Khan GK. Dermatology Life Quality Index (DLQI)--a simple practical measure for routine clinical use. Clin Exp Dermatol 1994;19:210-6.

28 Balp M-M, Vietri J, Tian $\mathrm{H}$, et al. The impact of chronic urticaria from the patient's perspective: a survey in five European countries. Patient 2015;8:551-8.

29 Tat TS. Higher levels of depression and anxiety in patients with chronic urticaria. Med Sci Monit 2019;25:115-20.

30 Engin B, Uguz F, Yilmaz E, et al. The levels of depression, anxiety and quality of life in patients with chronic idiopathic urticaria. $J$ Eur Acad Dermatol Venereol 2008;22:36-40.
31 Staubach P, Eckhardt-Henn A, Dechene M, et al. Quality of life in patients with chronic urticaria is differentially impaired and determined by psychiatric comorbidity. $\mathrm{Br} J$ Dermatol 2006;154:294-8.

32 Gimenéz-Arnau AM, Spector S, Antonova E, et al. Improvement of sleep in patients with chronic idiopathic/spontaneous urticaria treated with omalizumab: results of three randomized, double-blind, placebo-controlled studies. Clin Transl Allergy 2016;6:32.

33 He G-Y, Tsai T-F, Lin C-L, et al. Association between sleep disorders and subsequent chronic spontaneous urticaria development: a population-based cohort study. Medicine 2018;97:e11992.

34 Buysse DJ, Reynolds CF, Monk TH, et al. The Pittsburgh sleep quality index: a new instrument for psychiatric practice and research. Psychiatry Res 1989;28:193-213.

35 Jianli $\mathrm{C}$, Che J. The effect of acupuncture on serum IgE level in patients with chronic urticaria. J Tradit Chin Med 2006;26:189-90.

36 Gao H, Li X-Z, Ye W-W, et al. [Influence of penetrative needling of Shendao (GV 11) on the symptom score and serum IgE content in chronic urticaria patients]. Zhen Ci Yan Jiu 2009;34:272-5.

37 Shi Y, Zheng H, Zhou S, et al. Efficacy and safety of acupuncture for patients with chronic urticaria: study protocol of a randomized, sham-controlled pilot trial. Trials 2019;20:326.

38 Streitberger K, Kleinhenz J. Introducing a placebo needle into acupuncture research. Lancet 1998;352:364-5.

39 Lundeberg T, Lund I, Sing A, et al. Is placebo acupuncture what it is intended to be? Evid Based Complement Alternat Med 2011;2011:1-5.

40 Moffet HH. Sham acupuncture may be as efficacious as true acupuncture: a systematic review of clinical trials. $J$ Altern Complement Med 2009;15:213-6.

41 Zhao L, Chen J, Li Y, et al. The long-term effect of acupuncture for migraine prophylaxis: a randomized clinical trial. JAMA Intern Med 2017;177:508-15.

42 MacPherson H, Tilbrook H, Richmond S, et al. Alexander technique lessons or acupuncture sessions for persons with chronic neck pain: a randomized trial. Ann Intern Med 2015;163:653-62.

43 Ee C, Xue C, Chondros P, et al. Acupuncture for menopausal hot flashes: a randomized trial. Ann Intern Med 2016;164:146-54.

44 Jeon SW, Kim KS, Nam HJ. Long-Term effect of acupuncture for treatment of tinnitus: a randomized, patient- and assessor-blind, sham-acupuncture-controlled, pilot trial. J Altern Complement Med 2012;18:693-9

45 Moher D, Hopewell S, Schulz KF, et al. Consort 2010 explanation and elaboration: updated guidelines for reporting parallel group randomised trials. J Clin Epidemiol 2010;63:e1-37.

46 MacPherson H, Altman DG, Hammerschlag R, et al. Revised standards for reporting interventions in clinical trials of acupuncture (stricta): extending the consort statement. J Evid Based Med 2010;3:140-55. 